\title{
Care of community-dwelling older adults with dementia and their caregivers
}

\author{
Natalie Warrick MSc, Jeanette C. Prorok MSc, Dallas Seitz MD PhD
}

— Cite as: CMAJ 2018 July 3;190:E794-9. doi: 10.1503/cmaj.170920

CMAJ Podcasts: author interview at https://soundcloud.com/cmajpodcasts/170920-view

I

n Canada, about 500000 individuals are currently affected by dementia. ${ }^{1,2}$ Caring for people with dementia is complex and costs the health system $\$ 15$ billion annually. ${ }^{1,2}$ The findings of qualitative studies involving people living with dementia and their caregivers have suggested that improvements in care and health care for these individuals would be welcomed. ${ }^{3,4}$ Most individuals are first diagnosed and receive care for dementia in community or primary care settings; community-based services are the mainstay of support for people with dementia, with referral to specialists in dementia when indicated. ${ }^{5}$ The steering committee for the Third Canadian Consensus Conference on the Diagnosis and Treatment of Dementia recommended that all primary care providers have knowledge of local services, supports and models of care that enhance outcomes for people living with dementia and caregivers. ${ }^{6}$ We review clinical questions about community-based approaches to identifying and supporting older adults with dementia and their caregivers. We summarized evidence from meta-analyses, systematic reviews, randomized controlled trials (RCTs) and observational studies (Box 1).1,2,5-42

\section{Is earlier diagnosis of dementia in community and primary care settings beneficial?}

The findings of a 2011 study in England suggested substantial underdiagnosis of dementia in primary care. ${ }^{42}$ Identification of dementia is a necessary first step for accessing services. Individuals with dementia can be identified either through population-level screening or through targeted case finding; however, expert opinion does not support screening and instead favours case finding in primary care settings. ${ }^{9,10} \mathrm{Clin}$ ically, this means that evaluation for dementia should be started when there is a high probability of dementia based on the presence of risk factors (e.g., increased cardiovascular risk, or history of neurologic disorders such as stroke or Parkinson disease $)^{43}$ or when patients or caregivers raise concerns about cognition or functioning. ${ }^{5}$ Identifying dementia using case-finding approaches may result in reduced numbers of false-positives when compared with screening of asymp-

\section{KEY POINTS}

- Case-finding approaches that target individuals at high risk for dementia are suggested for the early detection of dementia in community and primary care settings.

- Multicomponent community programs, caregiver education and training, some respite programs and care management for dementia have been shown to delay admission to longterm care.

- Distress is common among caregivers for people with dementia and can be reduced by a variety of community supports and services.

- Referral of individuals to services that are provided by specialists in dementia in geriatric medicine, geriatric psychiatry or neurology are suggested primarily for the most clinically complex patients with dementia.

- All community-based health care providers should have knowledge of local supports and services available for people with dementia in their communities.

tomatic populations, according to a meta-analysis of cognitive tests. ${ }^{11,26}$ While there are no disease-modifying treatments available at this time, qualitative studies suggest that early identification can help relieve distress and uncertainty for people with dementia and their caregivers, ${ }^{3,4}$ facilitate discussions around future care planning, and promote earlier connection to community-based supports and programs. ${ }^{41}$ Detailed information on the prevention, evaluation and management of dementia are available in a series of clinical review articles that were based on the Canadian Consensus Conference on the Diagnosis and Treatment of Dementia. ${ }^{44-49}$ The key clinical processes to be followed in the evaluation of potential dementia include a careful history of the cognitive changes from the patient and family or caregiver (when available), evaluation for potential reversible causes of cognitive impairment through a review of medications and substances, completion of recommended screening bloodwork, and neuroimaging in specific circumstances, along with completion of cognitive screening tests (Box 2). ${ }^{50}$ 


\section{Can good community care help to delay admission to long-term care for older adults with dementia?}

According to the Alzheimer's Association in the United States, the primary goal of most people with dementia is to remain safely at home and delay admission to long-term care. ${ }^{51}$ Several communitybased interventions or supports have been shown to delay or prevent admission to long-term care, including multicomponent in-home and community support programs, education and training programs for caregivers, day programs and other forms of respite, and case management of dementia.

Multicomponent in-home and community programs may include elements of in-home assistance with personal care, coordination of dementia services and access to adult day programs. A meta-analysis of high-quality studies of multicomponent community-based interventions showed significant reductions in admissions to long-term care facilities, although the time to admission was not affected..$^{30}$ In 2005, an RCT that evaluated a combination of regularly scheduled in-home respite, caregiver education and family caregiver support group found a delay in admission to long-term care compared with usual care. ${ }^{27}$ However, a recent systematic review of multicomponent interventions did not find that these interventions had any effect on risk of admission to hospital, ${ }^{30}$ although the researchers observed a reduction in the number of days spent in hospital for those who were admitted. ${ }^{41}$

A meta-analysis of interventions for caregiver education showed a significant reduction in the risk of admission to longterm care and a longer time dwelling in the community compared with usual care, with a $12 \%-18 \%$ reduction in the proportion of individuals admitted to long-term care. ${ }^{36}$ One of the largest caregiver intervention studies is the New York University Caregiver Intervention program. ${ }^{27}$ This program included six sessions of individual and family counselling education (each session was one to three hours in duration), followed by weekly support groups and counsellor support as needed over the course of a year ${ }^{27}$ for community-dwelling individuals with dementia in an outpatient research clinic. The program was associated with a

\section{Box 1: Evidence used in this review}

We searched PsycINFO; Embase; Ovid MEDLINE; and relevant grey literature from Google Scholar; Canadian Institute for Health Information; Health Canada; Ontario Ministry of Health and LongTerm Care; and the Alzheimer Societies of Ontario and Canada for English-language evidence on interventions and models of care, for community-dwelling older adults with dementia and their caregivers. We used the search terms systematic review, metaanalysis, randomized controlled trial (RCT) AND dementia (OR synonyms) AND intervention/topic (i.e., respite, case finding) AND outcomes (e.g., time and rate of admission to long-term care, admission to hospital), and selected 40 articles for discussion in this review. Appendices 1-3 contain the search strategy, study flowchart and a detailed description of the review process, respectively (available at www.cmaj.ca/lookup/suppl/doi:10.1503/ cmaj.170920/-/DC1).
28.3\% (adjusted hazard ratio $0.72,95 \% \mathrm{Cl}$ [confidence interval] 0.54 to 0.96 ) reduction in the rate of transition to long-term care compared with usual care and, furthermore, delayed placement in long-term care by 329 days compared with usual care. ${ }^{52}$ The STrAtegies for RelaTives (START) coping strategy program for caregivers included a manual-based intervention for caregiver education that comprised eight one-hour sessions for caregivers, which was also found to be cost-effective. ${ }^{22}$

Some forms of respite programs have also been associated with reduced rates of admission to long-term care. A prospective,

\section{Box 2: Evaluation of dementia in older adults ${ }^{44-50}$}

History of cognitive and functional changes from patient and caregiver

- Onset and duration of cognitive changes

- Course of cognitive changes

- Review of symptoms related to memory, language, apraxia, and visuospatial and executive functioning

- Changes in abilities to perform basic and instrumental activities of daily living

Review of medical history

- Stroke, transient ischemic attack, Parkinson disease, seizures, head injury, hypertension, diabetes, dyslipidemia, atrial fibrillation, falls

Medication and substance review

- Medication compliance

- Medications with anticholinergic properties or sedating effects (e.g., opioids, anticonvulsants, antipsychotics, benzodiazepines, hypnotics)

- Substance misuse: alcohol, over-the-counter medications, marijuana

Completion of screening bloodwork

- Complete blood cell count, serum electrolytes, urea, calcium, thyroid-stimulating hormone, vitamin $\mathrm{B}_{12}$, fasting blood glucose

Indications for neuroimaging

- Young age (<60 yr), presence of localizing neurologic findings, atypical course, rapid progression, history of head trauma

Completion of cognitive screening tests

- Brief cognitive screening tests (< 10 min to complete)

- Mini-Cog: three-word recall and clock-drawing test

- General Practitioner assessment of Cognition (GPCOG): online evaluation of cognition (http://gpcog.com.au/)

- Memory Impairment Screen: four-word delayed free and category-cued recall task

- Longer cognitive screening tests (> 10 min to complete)

- Mini-Mental State Examination: if cognitive impairment is suspected to be of at least moderate severity

- Montreal Cognitive Assessment: evaluation of mild cognitive impairment or early possible dementia

- Evaluations reported by caregivers

- AD8 Dementia Screening Interview: eight-item questionnaire completed by caregivers about cognitive changes

- Informant Questionnaire on Cognitive Decline in the Elderly (IQCODE): informant questionnaire on cognitive changes, multiple versions 
nonrandomized study conducted in northern Sweden found reduced admission rates among users of day programs (i.e., the person with dementia attended an out-of-home program two to three times per week) compared with those who did not attend the day program. ${ }^{39}$ However, increased rates of placement in long-term care were reported in two before-and-after studies comparing patients who attended day programs and those who did not, although these results may have been confounded by functional disability and behavioural symptoms. ${ }^{20,24}$ The current evidence for the effect of institutional respite (e.g., short-stay overnight respite in long-term care) on permanent admissions to long-term care is unclear, with some indications that it may lead to higher rates of placement; ${ }^{37}$ however, longer-duration respite programs may be beneficial. ${ }^{35}$

A 2015 systematic review reported that case management was associated with reduced admissions to long-term care relative to usual care at six and eight months. ${ }^{32}$ Case management of dementia involves the use of standardized protocols for assessment, care management support and coordination of community services. In many regions of Canada, the First Link program provided by the Alzheimer Society of Canada engages primary care providers in actively referring the newly diagnosed and their caregivers to society resources, which may provide some of the components of case management for dementia. A prospective cohort study that compared self-referrals to society resources with referrals made through First Link found that primary care providers using First Link expedited patient access to the society 11 months earlier than usual referral methods. ${ }^{23}$

\section{What supports or services are available that can help to reduce caregiver stress?}

The Alzheimer's Association in the US reported that stress is common among caregivers of people with dementia; ${ }^{51}$ data from Canada show that about $30 \%$ report substantial distress. ${ }^{53} \mathrm{~A}$ systematic review found that caregiver stress is also associated with placement of patients in long-term care, ${ }^{54}$ and poor mental health and medical outcomes among caregivers themselves. ${ }^{51}$ Large effects for caregiver depression and self-esteem were observed in a systematic review of multicomponent community support programs. ${ }^{26}$ Reductions in caregiver burden and depression were reported in a meta-analysis of two trials of a caregiver support program. ${ }^{18}$ Case management of dementia has also been shown to improve caregiver outcomes. An RCT involving 408 patients with dementia and their caregivers in southern California provided education for primary care providers in the treatment and care of patients with dementia, and assigned each patient with dementia and their caregiver a care manager (primarily social workers). ${ }^{55}$ Care managers developed a problem list and assisted with addressing concerns about dementia care with one in-home meeting and follow-up telephone support as needed for at least 12 months. ${ }^{55}$ The trial showed significant improvements in the quality of care for patients with dementia that was provided in primary care and improved access to community services for the intervention group compared with usual care. A similar model of care management for dementia used advanced practice nurses as care managers in primary care settings. An RCT evaluated the effect of these care managers providing caregivers with nonpharmacologic recommendations for managing behavioural symptoms of dementia, along with coordination of community services for 12 months. ${ }^{14}$ Modest improvements in caregiver stress, as measured on the Neuropsychiatric Inventory Caregiver Distress Scale, were observed at 12 months for individuals receiving case management compared with controls (mean difference -2.2 , $95 \% \mathrm{Cl}-4.2$ to -0.2 ; effect size 0.3 ) but not at 18 months (differences in mean scores $-1.0,95 \% \mathrm{Cl}-3.0$ to 1.0 ; effect size 0.1 ). ${ }^{14}$

\section{When should primary care physicians refer patients to a specialist in dementia care?}

Some physician specialists, including geriatric medicine, geriatric psychiatry and neurology specialists, have enhanced training in the diagnosis and management of dementia. As of 2018, there were 334 geriatric medicine physicians, 233 geriatric psychiatrists and 1240 neurologists (a small number of whom would specialize in dementia care) who were certified by the Royal College of Physicians and Surgeons of Canada. ${ }^{56}$ As numbers of people affected by dementia in Canada increase, selective referral of patients to the limited number of specialists in dementia in Canada will be necessary. Yet a chart review of patients with dementia in urban Canadian primary care practices found that $82 \%$ of individuals with a diagnosis of dementia were referred to any specialist in dementia. ${ }^{31}$ The guideline from the Canadian Consensus Conference on the Diagnosis and Treatment of Dementia recommends referrals to specialists in dementia under specific circumstances, including diagnostic uncertainty, presence of substantial behavioural symptoms, challenges related to medications for dementia, assistance with complex clinical problems, genetic counselling, participation in research studies, or assistance with referrals to dementia programs or community services. ${ }^{29}$

Information on the effect of specialist care on dementiarelated outcomes remains limited primarily because of the small numbers of studies conducted to date. Intensive geriatric psychiatry case management and outreach programs have been associated with beneficial effects on caregiver stress, total caregiving time and reductions in risks associated with community-living for people compared with usual community-based services. ${ }^{16} \mathrm{~A}$ recent observational study that evaluated early referral to a specialist in dementia and risk of placement in long-term care found an increased likelihood of placment in long-term care among older adults with dementia who were referred to these specialists, although patients who are referred to specialists tend to have greater behavioural, neurologic and medical complexity, which may also increase their risk for placement in long-term care. ${ }^{57,58} \mathrm{An} \mathrm{RCT}$ that compared quality of care in primary care and memory clinics found no observable differences between these settings on patient outcomes for individuals who received their initial diagnosis in a specialty memory clinic..$^{59}$ Memory clinics based in primary care, where family physicians and a primary care team provide diagnostic evaluation and management of patients with dementia for a defined population, have recently emerged as a method to enhance access to dementia care in 
primary care and decrease unnecessary referrals to specialists in the treatment of dementia. ${ }^{40,60,61}$ In addition, these specialists are incorporated frequently into care management of dementia or primary care models of dementia care in a capacity enhancement and supportive role, which may also optimize access to specialists in the treatment of dementia compared with typical referral practices to specialty memory clinics. ${ }^{12}$

\section{Box 3: What are the evidence gaps?}

- For most community-based services for dementia, additional high-quality research is needed, particularly in the areas of respite programs.

- Randomized controlled trials that evaluate the effects of casefinding approaches for identifying older adults with dementia are required.

- Interventions that may reduce unnecessary admissions to hospital and use of emergency departments among older adults with dementia are not known at this time.

- The critical or necessary elements for the effect of multicomponent interventions on caregiver stress need further elaboration.

- The effects of specialists in dementia on outcomes in people with dementia and identification of groups of older adults who will have the greatest benefit from specialist involvement requires additional research.

\section{What are the uncertainties?}

There are several limitations and unanswered questions related to the organization of community-based dementia services, which require further research and evaluation (Box 3). In some regions of Canada, there may be limited access to some of the supports and services identified in our review. Much of the available evidence discussed here is derived from health systems outside Canada. Adaptations will likely be required in many Canadian jurisdictions. All health care providers should be aware of their local resources for dementia and which services may be most beneficial for their patients and caregivers, and there are several online resources available that may be helpful for clinicians, patients and caregivers (Table 1 ). ${ }^{62}$ Health system planners and policy-makers should be aware of services that have been shown to be beneficial for people and plan to meet the increasing demand for these services in the future.

\section{Conclusion}

The identification and care of community-dwelling people with dementia and their caregivers is complex and will often involve multiple supports and services to optimize outcomes. Existing guideline recommendations for dementia and high-quality evidence underscore that community-level multicomponent supports, including caregiver education and training programs,

Table 1: Resources for Alzheimer disease and dementia ${ }^{62}$

Organization

Website URL

Resource type

\section{Resources in Canada}

Alzheimer Society of Canada

Dementia guide

brainXchange

Murray Alzheimer Research and Education Program

Government of Canada - Care conversations, it's about you and the person you support

Canadian Consortium on Neurodegeneration in Aging

\section{International resources}

Alzheimer's Association

Alzheimer's Disease

International

Alzheimer's Foundation of America

Dementia Evidence Toolkit
alzheimer.ca/en/Home (English) alzheimer.ca/fr/Home (Français)

www.dementiaguide.com

www.brainxchange.ca/

https://uwaterloo.ca/murray-alzheimer -research-and-education-program/

www.canada.ca/en/employment-social -development/corporate/seniors/forum/ care-conversation.html

http://ccna-ccnv.ca/en/

www.alz.org/

www.alz.co.uk

www.alzfdn.ca

www.toolkit.modem-dementia.org.uk/
Resources for patients, caregivers and health care providers, and links to provincial and local dementia resources

Information about dementia and links to resources related to dementia for clinicians and caregivers

Links to resources on dementia and other neurologic disorders, webinars on topics related to dementia and online forums

Educational resources related to dementia, including the By Us For Us Guides created by patients and caregivers, and other resources for dementia

Includes information to help support families and friends in their caregiving roles

Information on the Canadian Consortium on Neurodegeneration in Aging research program about Alzheimer disease and related forms of dementia

Resources for patients and caregivers, and links to international services and resources

Links to international organizations for Alzheimer disease

Information for informal caregivers and health care professionals, and resources about caregiver training

Searchable database of interventions for people with dementia including nonpharmacologic and pharmacologic treatments 
some forms of respite programs and case management approaches for dementia, are effective in delaying admission to long-term care settings for older adults with dementia and reducing caregiver stress.

\section{References}

1. Mapping connections: an understanding of neurological conditions in Canada Protecting Canadians from illness. Ottawa: Public Health Agency of Canada; 2014. Available: www.phac-aspc.gc.ca/publicat/cd-mc/mc-ec/assets/pdf/mc-ec-eng.pdf (accessed 2017 Apr. 17).

2. Making the case for investing in mental health in Canada. Calgary: Mental Health Commission of Canada; 2011. Available: www.mentalhealthcommission.ca/ sites/default/files/2016-06/Investing_in_Mental_Health_FINAL_Version_ENG.pdf (accessed 2017 Apr. 17).

3. Prorok JC, Horgan S, Seitz DP. Health care experiences of people with dementia and their caregivers: a meta-ethnographic analysis of qualitative studies. CMAJ 2013;185:E669-80.

4. Prorok JC, Hussain M, Horgan S, et al. 'I shouldn't have had to push and fight': health care experiences of persons with dementia and their caregivers in primary care. Aging Ment Health 2017;21:797-804.

5. Assessing dementia: the Canadian consensus. Organizing Committee Canadian Consensus Conference on the Assessment of Dementia. CMAJ 1991;144:851-3.

6. Hogan DB, Bailey P, Carswell A, et al. Management of mild to moderate Alzheimer's disease and dementia. Alzheimers Dement 2007;3:355-84.

7. U.S. Preventive Services Task Force. Screening for dementia: recommendation and rationale. Ann Intern Med 2003;138:925-6.

8. Health Care in Canada, 2011: a focus on seniors and aging. Ottawa: Canadian Institute for Health Information; 2011. Available: https://secure.cihi.ca/free_products/ HCIC_2011_seniors_report_en.pdf (accessed 2018 Jan. 15).

9. Kurdyak P, Lin E, Green D, et al. Validation of a population-based algorithm to detect chronic psychotic illness. Can J Psychiatry 2015;60:362-8.

10. Wübbeler M, Thyrian JR, Michalowsky B, et al. How do people with dementia utilise primary care physicians and specialists within dementia networks? Results of the Dementia Networks in Germany (DemNet-D) study. Health Soc Care Community 2017;25:285-94.

11. Ashford JW, Borson S, O'Hara R, et al. Should older adults be screened for dementia? It is important to screen for evidence of dementia! Alzheimers Dement 2007;3:75-80.

12. Borson S, Chodosh J. Developing dementia-capable health care systems: a 12-step program. Clin Geriatr Med 2014;30:395-420.

13. Boustani M, Peterson B, Hanson L, et al.; U.S. Preventive Services Task Force. Screening for dementia in primary care: a summary of the evidence for the U.S. Preventive Services Task Force. Ann Intern Med 2003;138:927-37.

14. Callahan CM, Boustani MA, Unverzagt FW, et al. Effectiveness of collaborative care for older adults with Alzheimer disease in primary care: a randomized controlled trial. JAMA 2006;295:2148-57.

15. Pottie K, Rahal R, Jaramillo A, et al.; Canadian Task Force on Preventive Health Care. Recommendations on screening for cognitive impairment in older adults. CMAJ 2016;188:37-46.

16. Challis $D$, von Abendorff R, Brown $P$, et al. Care management, dementia care and specialist mental health services: an evaluation. Int J Geriatr Psychiatry 2002; 17:315-25.

17. Chiu M, Pauley T, Wesson V, et al. Evaluation of a problem-solving (PS) techniques-based intervention for informal carers of patients with dementia receiving in-home care. Int Psychogeriatr 2015;27:937-48.

18. Jensen M, Agbata IN, Canavan M, et al. Effectiveness of educational interventions for informal caregivers of individuals with dementia residing in the community: systematic review and meta-analysis of randomised controlled trials. Int J Geriatr Psychiatry 2015;30:130-43.

19. Knapp M, King D, Romeo R, et al. Cost effectiveness of a manual based coping strategy programme in promoting the mental health of family carers of people with dementia (the START (STrAtegies for RelaTives) study): a pragmatic randomised controlled trial. BMJ 2013;347:f6342.

20. Kuzuya M, Izawa S, Enoki H, et al. Day-care service use is a risk factor for longterm care placement in community-dwelling dependent elderly. Geriatr Gerontol Int 2012;12:322-9.
21. Lee H, Cameron MH. Respite care for people with dementia and their carers. Cochrane Database Syst Rev 2004;(2):CD004396.

22. Livingston G, Barber J, Rapaport P, et al. Clinical effectiveness of a manual based coping strategy programme (START, STrAtegies for RelaTives) in promoting the mental health of carers of family members with dementia: pragmatic randomised controlled trial. BMJ 2013;347:f6276

23. McAiney CA, Hillier LM, Stolee P, et al. 'Throwing a lifeline': the role of First Link $^{\top \mathrm{M}}$ in enhancing support for individuals with dementia and their caregivers. Neurodegener Dis Manag 2012;2:623-38.

24. McCann JJ, Hebert LE, Li Y, et al. The effect of adult day care services on time to nursing home placement in older adults with Alzheimer's disease. Gerontologist 2005;45:754-63.

25. Meeuwsen E, Melis R, van der Aa G, et al. Cost-effectiveness of one year dementia follow-up care by memory clinics or general practitioners: economic evaluation of a randomised controlled trial. PLoS One 2013;8:e79797.

26. Mitchell AJ, Malladi S. Screening and case-finding tools for the detection of dementia. Part II: evidence-based meta-analysis of single-domain tests. Am J Geriatr Psychiatry 2010;18:783-800.

27. Mittelman MS, Ferris SH, Shulman E, et al. A family intervention to delay nursing home placement of patients with Alzheimer disease: a randomized controlled trial. JAMA 1996;276:1725-31.

28. Nyweide DJ, Anthony DL, Bynum JP, et al. Continuity of care and the risk of preventable hospitalization in older adults. JAMA Intern Med 2013;173:1879-85.

29. Patterson CJ, Gauthier S, Bergman H, et al. The recognition, assessment and management of dementing disorders: conclusions from the Canadian Consensus Conference on Dementia. CMAJ 1999;160(Suppl):S1-15.

30. Phelan EA, Debnam KJ, Anderson LA, et al. A systematic review of intervention studies to prevent hospitalizations of community-dwelling older adults with dementia. Med Care 2015;53:207-13.

31. Pimlott NJ, Siegel K, Persaud M, et al. Management of dementia by family physicians in academic settings. Can Fam Physician 2006;52:1108-9.

32. Reilly S, Miranda-Castillo C, Malouf R, et al. Case management approaches to home support for people with dementia. Cochrane Database Syst Rev 2015;1: CD008345.

33. Schulz R, Lustig A, Handler S, et al. Technology-based caregiver intervention research: Current status and future directions. Gerontechnology (Valkenswaard) 2002;2:15-47. doi:10.4017/gt.2002.02.01.003.00.

34. Smits $\mathrm{CH}$, de Lange J, Dröes RM, et al. Effects of combined intervention programmes for people with dementia living at home and their caregivers: a systematic review. Int J Geriatr Psychiatry 2007;22:1181-93.

35. Sörensen S, Pinquart M, Duberstein P. How effective are interventions with caregivers? An updated meta-analysis. Gerontologist 2002;42:356-72.

36. Spijker A, Vernooij-Dassen M, Vasse E, et al. Effectiveness of nonpharmacological interventions in delaying the institutionalization of patients with dementia: a meta-analysis. J Am Geriatr Soc 2008;56:1116-28.

37. Vandepitte S, Van Den Noortgate N, Putman K, et al. Effectiveness of respite care in supporting informal caregivers of persons with dementia: a systematic review. Int J Geriatr Psychiatry 2016;31:1277-88.

38. Wilson JMG, Jungner G. Principles and practice of screening for disease. Geneva: World Health Organization; 1968.

39. Wimo A, Mattsson B, Adolfsson R, et al. Dementia day care and its effects on symptoms and institutionalization - a controlled Swedish study. Scand J Prim Health Care 1993;11:117-23.

40. Wolfs CA, Dirksen CD, Kessels A, et al. Economic evaluation of an integrated diagnostic approach for psychogeriatric patients: results of a randomized controlled trial. Arch Gen Psychiatry 2009;66:313-23.

41. Zabalegui A, Hamers JP, Karlsson S, et al. Best practices interventions to improve quality of care of people with dementia living at home. Patient Educ Couns 2014;95:175-84.

42. Connolly A, Gaehl E, Martin H, et al. Underdiagnosis of dementia in primary care: variations in the observed prevalence and comparisons to the expected prevalence. Aging Ment Health 2011;15:978-84.

43. Bamford C, Eccles M, Steen N, et al. Can primary care record review facilitate earlier diagnosis of dementia? Fam Pract 2007;24:108-16.

44. Patterson C, Feightner JW, Garcia A, et al. Diagnosis and treatment of dementia: 1 . Risk assessment and primary prevention of Alzheimer disease. CMAJ 2008;178:548-56. 
45. Feldman HH, Jacova C, Robillard A, et al. Diagnosis and treatment of dementia: 2. Diagnosis. CMAJ 2008;178:825-36.

46. Chertkow H, Massoud F, Nasreddine Z, et al. Diagnosis and treatment of dementia: 3. Mild cognitive impairment and cognitive impairment without dementia. CMAJ 2008;178:1273-85.

47. Hogan DB, Bailey P, Black S, et al. Diagnosis and treatment of dementia: 4. Approach to management of mild to moderate dementia. CMAJ 2008;179: 787-93.

48. Hogan DB, Bailey P, Black S, et al. Diagnosis and treatment of dementia: 5 . Nonpharmacologic and pharmacologic therapy for mild to moderate dementia. CMAJ 2008;179:1019-26.

49. Herrmann N, Gauthier S. Diagnosis and treatment of dementia: 6. Management of severe Alzheimer disease. CMAJ 2008;179:1279-87.

50. Cordell CB, Borson S, Boustani M, et al. Medicare Detection of Cognitive Impairment Workgroup. Alzheimer's Association recommendations for operationalizing the detection of cognitive impairment during the Medicare Annual Wellness Visit in a primary care setting. Alzheimers Dement 2013;9: 141-50.

51. Alzheimer's Association. 2017 Alzheimer's disease facts and figures. Alzheimers Dement 2017;13:325-73.

52. Mittelman MS, Haley WE, Clay OJ, et al. Improving caregiver well-being delays nursing home placement of patients with Alzheimer disease. Neurology 2006;67:1592-9.

53. Supporting informal caregivers - The heart of home care [executive summary]. Ottawa: Canadian Institute for Health Information; 2010.
54. Cepoiu-Martin M, Tam-Tham H, Patten S, et al. Predictors of long-term care placement in persons with dementia: a systematic review and meta-analysis. Int J Geriatr Psychiatry 2016;31:1151-71.

55. Vickrey BG, Mittman BS, Connor KI, et al. The effect of a disease management intervention on quality and outcomes of dementia care: a randomized, controlled trial. Ann Intern Med 2006;145:713-26.

56. The Royal College directory. Ottawa: Royal College of Physicians and Surgeons of Canada; 2018. Available: www.royalcollege.ca/rcdir/faces/home?_afrLoop $=31422562021454702 \& \_$afrWindowMode=0\&_adf.ctrl-state=1djv1mkizx_4 (accessed 2018 Mar. 1).

57. Pimouguet C, Le-Goff M, Rizzuto D, et al. Effect of early referral to specialist in dementia on institutionalization and functional decline: findings from a populationbased study. J Alzheimers Dis 2016;49:819-28.

58. Pimouguet C, Lavaud T, Dartigues J, et al. Dementia case management effectiveness on health care costs and resource utilization: a systematic review of randomized controlled trials. J Nutr Health Aging 2010;14:669-76.

59. Meeuwsen EJ, Melis RJ, Van Der Aa GC, et al. Effectiveness of dementia followup care by memory clinics or general practitioners: randomised controlled trial. BMJ 2012;344:e3086.

60. Wolfs CA, Kessels A, Dirksen CD, et al. Integrated multidisciplinary diagnostic approach for dementia care: randomised controlled trial. $\mathrm{Br} \mathrm{J}$ Psychiatry 2008;192:300-5.

61. Lee L, Hillier LM, Stolee P, et al. Enhancing dementia care: a primary carebased memory clinic. J Am Geriatr Soc 2010;58:2197-204.

62. Dillon WA, Prorok JC, Seitz DP. Content and quality of information provided on Canadian dementia websites. Can Geriatr J 2013;16:6-15.
Competing interests: Dallas Seitz participated in clinical trials of Alzheimer disease that were sponsored by F. Hoffmann-La Roche. No other competing interests were declared.

This article has been peer reviewed.

Affiliations: Cancer Care Ontario (Warrick, Prorok, Seitz); Institute of Health Policy, Management and Evaluation (Warrick), University of Toronto, Toronto, Ont.; School of Public Health and Health Systems (Prorok), University of Waterloo, Waterloo, Ont.; Department of Psychiatry (Seitz), Queen's University, Kingston, Ont.

Contributors: All authors contributed to the writing of the manuscript, and quality assessment and data analysis. Natalie Warrick and Dallas Seitz conceived the idea and co-led the overall development of the paper. Natalie Warrick was primarily responsible for database searches, and Jeanette Prorok was primarily responsible for data extraction. All of the authors revised the manuscript critically for important intellectual content, gave final approval of the version to be published and agreed to be accountable for all aspects of the work.

Funding: This preparation of this manuscript was undertaken while all authors were employed at Cancer Care Ontario, as part of the Dementia Capacity Planning project funded by the Ontario Ministry of Health and Long-Term Care. Natalie Warrick was supported by doctoral awards provided by the Alzheimer Society of Canada Research Program, and an Ontario Graduate Scholarship and a fellowship through the Institute of Health Policy, Management and Evaluation, University of Toronto. Jeanette Prorok was supported by a Doctoral Research Award from CIHR. Dallas Seitz receives funding from the Canadian Consortium on Neurodegeneration and Aging (funded by the Canadian
Institutes of Health Research [CIHR]), the Ontario Brain Institute and Cancer Care Ontario.

Acknowledgements: The authors thank the members of the Dementia Capacity Planning team, including Marnie McKinnon (Cancer Care Ontario [CCO]), Esti Heale (CCO), Kerry Allerton (formerly of $\mathrm{CCO}$ ), Magda Nunes de Melo of the (Institute of Clinical and Evaluative Sciences), Kirk Nylen (Ontario Brain Institute $[\mathrm{OBI}])$, Jenine Paul (OBI), Graham Woodward (CorHealth Ontario), Michael Robertson (Ontario Ministry of Health and Long-Term Care [MOHLTC]), Katie O'Connell (MOHLTC), Dilini Urukulaarachchi (MOHLTC) and Mike O'Campo (MOHLTC). The authors would also like to thank Roxanne Leung for her assistance with the preparation of this manuscript.

Correspondence to: Dallas Seitz, seitzd@ providencecare.ca 